\title{
Multicritical Point Relations in Three Dual Pairs of Hierarchical-Lattice Ising Spin-Glasses
}

\author{
Michael Hinczewski and A. Nihat Berker \\ Department of Physics, Istanbul Technical University, Maslak 34469, Istanbul, Turkey, \\ Department of Physics, Massachusetts Institute of Technology, Cambridge, Massachusetts 02139, U.S.A., and \\ Feza Gürsey Research Institute, TÜBITAK - Bosphorus University, Cengelköy 34680, Istanbul, Turkey
}

\begin{abstract}
The Ising spin-glasses are investigated on three dual pairs of hierarchical lattices, using exact renormalization-group transformation of the quenched bond probability distribution. The goal is to investigate a recent conjecture which relates, on such pairs of dual lattices, the locations of the multicritical points, which occur on the Nishimori symmetry line. Towards this end we precisely determine the global phase diagrams for these six hierarchical spin-glasses, using up to $2.5 \times 10^{9}$ probability bins to represent the quenched distribution subjected to an exact renormalization-group transformation. We find in all three cases that the conjecture is realized to a very good approximation, even when the mutually dual models belong to different spatial dimensionalities $d$ and have different phase diagram topologies at the multicritical points of the conjecture and even though the contributions to the conjecture from each lattice of the dual pair are strongly asymmetric. In all six phase diagrams, we find reentrance near the multicritical point. In the models with $d=2$ or 1.5 , the spin-glass phase does not occur and the phase boundary between the ferromagnetic and paramagnetic phases is second order with a strong violation of universality.

PACS numbers: 75.10.Nr, 64.60.Kw, 05.45.Df, 05.10.Cc
\end{abstract}

\section{INTRODUCTION}

The phase diagram structure of spin-glasses remains an open field of inquiry, since most approaches to the problem rely on approximations. Any exact analytical result in this area is thus very valuable, both for the direct information it provides and as a test for approximation methods. Over the last few years striking progress has been made combining the replica method, duality, and symmetry arguments 1, 2, 3, 4, 5], an approach which has yielded the exact locations of the multicritical points in the Ising and Potts spin-glasses on the square lattice and in the four-dimensional random-plaquette gauge model. The most recent result in this series [5] is a general conjecture relating the multicritical point locations of any spin-glasses on a pair of mutually dual lattices. In support of the conjecture, estimates based on Monte Carlo simulations were given for Ising spin-glasses, in $d=2$, on the dual pairs of triangular and hexagonal lattices and, in $d=3$, on the dual pairs of bilinear and latticegauge interactions on the cubic lattice. In both cases, within the numerical limitations, the conjecture is approximately satisfied.

We propose here to extensively test the conjecture in an alternative fashion using hierarchical lattices [6, 7, 8], by looking at Ising spin-glasses on mutually dual pairs [9, 10, 11, 12] of such lattices. These constitute ideal testing grounds, since an exact renormalization-group transformation for the quenched bond probability distribution can be constructed for such lattices, yielding global phase diagrams and critical properties. Accordingly, the location of the phase boundaries and of the multicritical points are precisely determined. We thus investigate three pairs of hierarchical lattices, and in the end find that the conjecture is very nearly satisfied for all of them.

\section{THE CONJECTURE}

The Ising spin-glass is given by the Hamiltonian

$$
-\beta H=\sum_{\langle i j\rangle} J_{i j} s_{i} s_{j}
$$

where $s_{i}= \pm 1$ at each site $i,\langle i j\rangle$ denotes a sum over nearest-neighbor pairs of sites, and the bond strengths $J_{i j}$ are equal to $+J$ with probability $1-p$ and $-J$ with probability $p$. The limits $p=0$ and $p=1$ correspond to purely ferromagnetic and purely antiferromagnetic systems respectively.

To give a brief overview of the conjecture, let us consider the model on an arbitrary lattice, and treat the randomness through the replica method, where the system is replicated $n$ times and the $n \rightarrow 0$ limit is eventually taken, in order to get results for the physical system. The partition function of the $n$-replicated system after averaging over randomness, $Z_{n}$, can be expressed entirely as a function of $n+1$ "averaged" combinations of edge Boltzmann factors, $e^{ \pm J}$, associated with nearestneighbor bonds [3, 4]. These averaged Boltzmann factors, $x_{k}(p, J), k=0, \ldots, n$, have the form

$$
x_{k}(p, J)=p e^{-(n-k) J} e^{k J}+(1-p) e^{(n-k) J} e^{-k J},
$$

where the $k$ th factor corresponds to a configuration with a parallel-spin bond in $n-k$ replicas and an antiparallelspin bond in $k$ replicas [5]. Thus,

$$
Z_{n}=Z_{n}\left(x_{0}(p, J), x_{1}(p, J), \ldots, x_{n}(p, J)\right) .
$$

The partition function on the dual lattice, $Z_{n}^{*}$, can be expressed in a similar form,

$$
Z_{n}^{*}=Z_{n}^{*}\left(x_{0}^{*}(p, J), x_{1}^{*}(p, J), \ldots, x_{n}^{*}(p, J)\right),
$$


with the dual counterparts to the averaged Boltzmann factors given by

$$
\begin{aligned}
x_{2 k}^{*}(p, J)= & \left(\frac{e^{-J}+e^{J}}{\sqrt{2}}\right)^{n-2 k}\left(\frac{e^{-J}-e^{J}}{\sqrt{2}}\right)^{2 k}, \\
x_{2 k+1}^{*}(p, J)=(2 p-1)\left(\frac{e^{-J}+e^{J}}{\sqrt{2}}\right)^{n-2 k-1} & \\
& \cdot\left(\frac{e^{-J}-e^{J}}{\sqrt{2}}\right)^{2 k+1},
\end{aligned}
$$

for $0 \leq 2 k<2 k+1 \leq n$. $Z_{n}$ and $Z_{n}^{*}$ are related as [5]

$$
\begin{aligned}
Z_{n}\left(x_{0}(p, J), \ldots,\right. & \left.x_{n}(p, J)\right) \\
& =2^{a} Z_{n}^{*}\left(x_{0}^{*}(p, J), \ldots, x_{n}^{*}(p, J)\right),
\end{aligned}
$$

where $a$ is a constant, which can be eliminated by using Eq. (6) evaluated at two different sets of parameters, $\left(p_{1}, J_{1}\right)$ and $\left(p_{2}, J_{2}\right)$, giving a relationship of the form

$$
\begin{gathered}
Z_{n}\left(x_{0}\left(p_{1}, J_{1}\right), \ldots, x_{n}\left(p_{1}, J_{1}\right)\right) \\
\cdot Z_{n}^{*}\left(x_{0}\left(p_{2}, J_{2}\right), \ldots, x_{n}\left(p_{2}, J_{2}\right)\right) \\
=Z_{n}^{*}\left(x_{0}^{*}\left(p_{1}, J_{1}\right), \ldots, x_{n}^{*}\left(p_{1}, J_{1}\right)\right) \\
\cdot Z_{n}\left(x_{0}^{*}\left(p_{2}, J_{2}\right), \ldots, x_{n}^{*}\left(p_{2}, J_{2}\right)\right) .
\end{gathered}
$$

The individual partition functions $Z_{n}$ can be rewritten by extracting $x_{0}$, the averaged Boltzmann factor corresponding to an all-parallel spin state, thus effectively measuring the energy of the system relative to this state [3] :

$$
Z_{n}\left(x_{0}, x_{1}, \ldots, x_{n}\right)=x_{0}^{N_{B}} \mathcal{Z}_{n}\left(u_{1}, u_{2}, \ldots, u_{n}\right),
$$

where $N_{B}$ is the number of bonds in the lattice, and the reduced variables are $u_{i} \equiv x_{i} / x_{0}$. Eq. (7) becomes

$$
\begin{aligned}
& {[}\left.x_{0}\left(p_{1}, J_{1}\right) x_{0}\left(p_{2}, J_{2}\right)\right]^{N_{B}} \mathcal{Z}_{n}\left(u_{1}\left(p_{1}, J_{1}\right), \ldots, u_{n}\left(p_{1}, J_{1}\right)\right) \\
& \cdot \mathcal{Z}_{n}^{*}\left(u_{1}\left(p_{2}, J_{2}\right), \ldots, u_{n}\left(p_{2}, J_{2}\right)\right) \\
&=\left[x_{0}^{*}\left(p_{1}, J_{1}\right) x_{0}^{*}\left(p_{2}, J_{2}\right)\right]^{N_{B}} \mathcal{Z}_{n}^{*}\left(u_{1}^{*}\left(p_{1}, J_{1}\right), \ldots, u_{n}^{*}\left(p_{1}, J_{1}\right)\right) \\
& \quad \cdot \mathcal{Z}_{n}\left(u_{1}^{*}\left(p_{2}, J_{2}\right), \ldots, u_{n}^{*}\left(p_{2}, J_{2}\right)\right) .
\end{aligned}
$$

In general, the form of Eq. (9) is too complicated to yield useful information relating the locations of phase transitions. However, the multicritical points in both original and dual systems are expected to lie [13, 14, 15] on the Nishimori line 1], which simplifies the relation. Furthermore, the conjecture advanced in Ref. [5] states that, for the multicritical points $\left(p_{1 m}, J_{1 m}\right)$ of the original system and $\left(p_{2 m}, J_{2 m}\right)$ of its dual, Eq. (9) is satisfied when the leading Boltzmann factors $x_{0}$ from each side are equal,

$x_{0}\left(p_{1 m}, J_{1 m}\right) x_{0}\left(p_{2 m}, J_{2 m}\right)=x_{0}^{*}\left(p_{1 m}, J_{1 m}\right) x_{0}^{*}\left(p_{2 m}, J_{2 m}\right)$.

Since $\left(p_{1 m}, J_{1 m}\right)$ and $\left(p_{2 m}, J_{2 m}\right)$ lie on the Nishimori line,

$$
e^{2 J_{1 m}}=\frac{1-p_{1 m}}{p_{1 m}}, \quad e^{2 J_{2 m}}=\frac{1-p_{2 m}}{p_{2 m}} .
$$

From Eqs. (2) and (5), Eq. (10) gives

$$
\left(p_{1 m}^{n+1}+\left(1-p_{1 m}\right)^{n+1}\right)\left(p_{2 m}^{n+1}+\left(1-p_{2 m}\right)^{n+1}\right)=2^{-n} .
$$

Finally taking the limit, $n \rightarrow 0$, one obtains the condition

$$
H\left(p_{1 m}\right)+H\left(p_{2 m}\right)=1
$$

where $H(p) \equiv-p \log _{2} p-(1-p) \log _{2}(1-p)$. As expressed in Eq. (13), the conjecture is asserted to hold for multicritical points of Ising spin-glasses on any pair of mutually dual lattices [5].

\section{THE MULTITUDE OF ISING SPIN-GLASSES ON HIERARCHICAL LATTICES}

Hierarchical lattices $[6,7,8]$ are constructed by replacing every single bond, in a connected cluster of bonds, with the connected cluster of bonds itself, and repeating this step an infinite number of times. These provide models exactly solvable by renormalization group, with which complex problems have been studied and understood. For example, frustrated [16], spin-glass [17], randombond [18] and random-field [19], Schrödinger equation [20], lattice-vibration [21], dynamic scaling 22], aperiodic magnet 23], complex phase diagram 24], and directedpath 25] systems, etc., have been solved on hierarchical lattices.

To test the conjecture of Eq. (13), we study Ising spinglasses on the dual pairs of hierarchical lattices, depicted in Figs. 1] 2] and 3] Each lattice in a given pair is the dual of the other. These particular choice of lattices are motivated by their properties under renormalization-group transformation as related to physical lattices. The hierarchical lattices of Fig. 1(a) and (b) yield the two variants of the Migdal-Kadanoff recursion relations [26, 27] for dimension $d=2$ with length rescaling factor $b=3$. Similarly, the lattice in Fig. 2(a) yields a Migdal-Kadanoff recursion relation for $d=3, b=3$. Its dual lattice in Fig. 2(b) has $d=1.5, b=9$. (The two variants of the Migdal-Kadanoff recursion relations correspond to mutually dual hierarchical lattices only in $d=2$.) Lastly, the hybrid lattice in Fig. 3(a) is interesting because it has been shown to give very accurate results for the critical temperatures of the $d=3$ isotropic and anisotropic Ising model 28]. This lattice has $d=3, b=3$, while its dual in Fig. 3(b) has $d=1.5, b=9$.

\section{EXACT RENORMALIZATION-GROUP TRANSFORMATION OF HIERARCHICAL SPIN-GLASSES}

For a pure system, the renormalization-group transformation on a hierarchical lattice consists of a decimation by summing over the internal sites in each of the connected clusters making up the lattice (the right-hand 
(a)

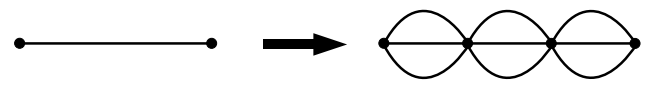

(b)

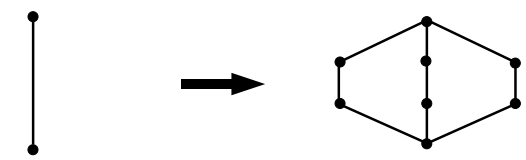

FIG. 1: The pair of mutually dual hierarchical lattices on which the $d=2, b=3$ Migdal-Kadanoff recursion relations are exact.

(a)

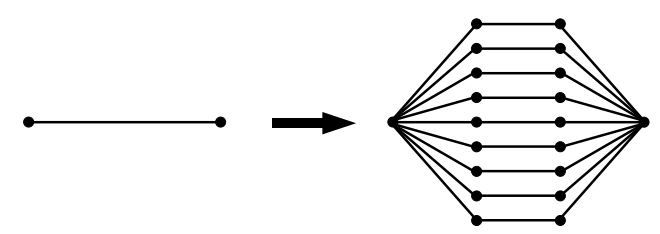

(b)
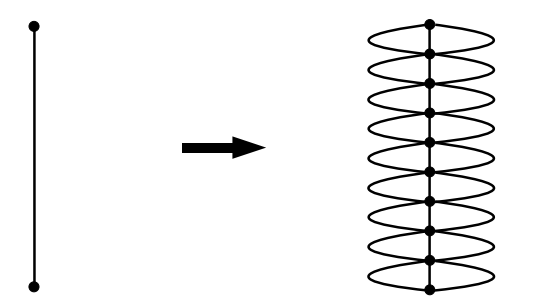

FIG. 2: Another pair of mutually dual hierarchical lattices. The Migdal-Kadanoff recursion relations are exact for lattice (a) with $d=3, b=3$. Its dual lattice, in (b), has $d=1.5$, $b=9$.

sides of Figs. 131). Thus, the hierarchical lattice construction process is reversed, as each connected cluster is replaced by a single renormalized bond. The decimation can be expressed as a mapping,

$$
J_{i^{\prime} j^{\prime}}^{\prime}=R\left(\left\{J_{i j}\right\}\right),
$$

where the set $\left\{J_{i j}\right\}$ are all the bonds within the connected cluster of the original system and $J_{i^{\prime} j^{\prime}}^{\prime}$ is the renormalized bond between sites $i^{\prime}$ and $j^{\prime}$ of the rescaled system. In the pure case, all $J_{i j}$ bonds are independent for $i j$, and the implementation of Eq. (14) is straightforward.

When quenched randomness is added to the system, the renormalization-group transformation is expressed in terms of quenched probability distributions [18], where the quenched probability distribution $\mathcal{P}^{\prime}\left(J_{i^{\prime} j^{\prime}}^{\prime}\right)$ in the rescaled system is calculated from $\mathcal{P}\left(J_{i j}\right)$ in the original system through the convolution

$$
\mathcal{P}^{\prime}\left(J_{i^{\prime} j^{\prime}}^{\prime}\right)=\int\left[\prod_{i j}^{i^{\prime} j^{\prime}} d J_{i j} \mathcal{P}\left(J_{i j}\right)\right] \delta\left(J_{i^{\prime} j^{\prime}}^{\prime}-R\left(\left\{J_{i j}\right\}\right)\right) .
$$

Here the product runs over all the bonds $i j$ in the connected cluster of the original system between sites $i^{\prime}$ and $j^{\prime}$. (a)

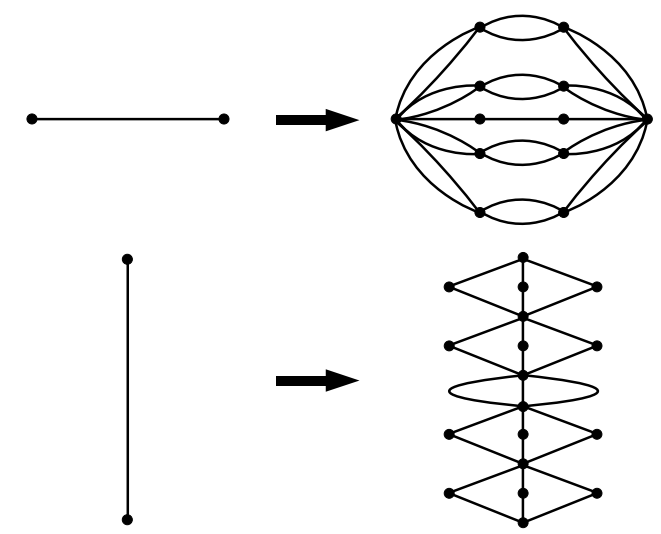

FIG. 3: Another pair of mutually dual hierarchical lattices, with $d=3, b=3$ and $d=1.5, b=9$ respectively.

The recursion of the quenched probability distribution, Eq. (15), is implemented numerically. The probability distribution is represented by histograms, each histogram being specified by a bond strength and an associated probability. Thus, for the spin-glass problem, the starting distribution consists of two histograms, one at $J$ with probability $1-p$, and one at $-J$ with probability $p$. Eq. (15) dictates the convolution of 9 probability distributions for the lattices of Fig. 1 and the convolution of 27 distributions for the lattices of Figs. 2]and 3 In this task, computational storage limits can be maximally exploited by factorizing Eq. (15) into an equivalent series of pairwise convolutions, each of which involves only two distributions convoluted using an appropriate $R$ function. The types of pairwise convolutions needed are a "bondmoving" convolution, with

$$
R_{\mathrm{bm}}\left(J_{i_{1} j_{1}}, J_{i_{2} j_{2}}\right)=J_{i_{1} j_{1}}+J_{i_{2} j_{2}},
$$

and a decimation convolution, with

$$
R_{\mathrm{dc}}\left(J_{i_{1} j_{1}}, J_{i_{2} j_{2}}\right)=\frac{1}{2} \ln \left[\frac{\cosh \left(J_{i_{1} j_{1}}+J_{i_{2} j_{2}}\right)}{\cosh \left(J_{i_{1} j_{1}}-J_{i_{2} j_{2}}\right)}\right],
$$

which is just the standard decimation transformation for a two-bond Ising segment.

Consider the hierarchical lattice in Fig. 1(a). If $\mathcal{P}_{\text {init }}$ is the initial probability distribution, a series of pairwise convolutions which yields the total convolution of Eq. (15) for this lattice is: (i) a bond-moving convolution of $\mathcal{P}_{\text {init }}$ with itself, yielding $\mathcal{P}_{1}$; (ii) a bond-moving convolution of $\mathcal{P}_{1}$ with $\mathcal{P}_{\text {init }}$, yielding $\mathcal{P}_{2}$; (iii) a decimation convolution of $\mathcal{P}_{2}$ with itself, yielding $\mathcal{P}_{3}$; (iv) a decimation convolution of $\mathcal{P}_{3}$ with $\mathcal{P}_{2}$, yielding $\mathcal{P}_{\text {final }}$. For the lattice in Fig. 2(a), the series is: (i) a decimation convolution of $\mathcal{P}_{\text {init }}$ with itself, yielding $\mathcal{P}_{1}$; (ii) a decimation convolution of $\mathcal{P}_{1}$ with $\mathcal{P}_{\text {init }}$, yielding $\mathcal{P}_{2}$; (iii) a bondmoving convolution of $\mathcal{P}_{2}$ with itself, yielding $\mathcal{P}_{3}$; (iv) a bond-moving convolution of $\mathcal{P}_{3}$ with itself, yielding $\mathcal{P}_{4}$; (v) a bond-moving convolution of $\mathcal{P}_{4}$ with itself, yielding $\mathcal{P}_{5}$; (vi) a bond-moving convolution of $\mathcal{P}_{5}$ with $\mathcal{P}_{2}$, yielding $\mathcal{P}_{\text {final }}$. For the lattice in Fig. B(a), the series is: (i) a 
bond-moving convolution of $\mathcal{P}_{\text {init }}$ with itself, yielding $\mathcal{P}_{1}$; (ii) a decimation convolution of $\mathcal{P}_{1}$ with itself, yielding $\mathcal{P}_{2}$; (iii) a decimation convolution of $\mathcal{P}_{2}$ with $\mathcal{P}_{1}$, yielding $\mathcal{P}_{3}$; (iv) a bond-moving convolution of $\mathcal{P}_{3}$ with itself, yielding $\mathcal{P}_{4} ;(\mathrm{v})$ a bond-moving convolution of $\mathcal{P}_{4}$ with itself, yielding $\mathcal{P}_{5} ;$ (vi) a decimation convolution of $\mathcal{P}_{\text {init }}$ with itself, yielding $\mathcal{P}_{6}$; (vii) a decimation convolution of $\mathcal{P}_{6}$ with $\mathcal{P}_{\text {init }}$, yielding $\mathcal{P}_{7}$; (viii) a bond-moving convolution of $\mathcal{P}_{7}$ with $\mathcal{P}_{5}$, yielding $\mathcal{P}_{\text {final }}$. As for the dual lattices in Figs. 1(b), 2 (b), and 3(b), the series of pairwise convolutions are identical to their counterparts above, except that each bond-moving is replaced by a decimation, and vice versa.

Since the number of histograms that constitute the probability distribution increases rapidly with each renormalization iteration, a binning procedure is used when the desired (large, namely up to $2.5 \times 10^{9}$ ) number of histograms is reached: Before every pairwise convolution, the histograms are placed on a grid, and all histograms falling into the same grid cell are combined into a single histogram in such a way that the average and the standard deviation of the probability distribution are preserved. Histograms falling outside the grid, representing a negligible part of the total probability, are similarly combined into a single histogram. Any histogram within a small neighborhood of a cell boundary is proportionately shared between the adjacent cells. In the current study, the binning procedure is done separately for $J>0$ and $J<0$. After the convolution, the original number of histograms is reattained.

In the current study, 40,000 bins are generally used, representing the renormalization-group flows of 80,000 variables, requiring the calculation of 40,000 local renormalization-group transformations at each renormalization-group iteration. The numerical results converge rapidly with increasing bin number. For maximal accuracy in determining the exact locations of the multicritical points, in the immediate vicinity of these points we used at least 1,000,000 histograms, representing the renormalization-group flows of 2,000,000 variables, requiring the calculation of 1,000,000 local renormalization-group transformations at each renormalization-group iteration. It should thus be noted that our analysis is an exact numerical solution of Ising spin-glasses on hierarchical lattices.

\section{RESULTS}

Global phase diagrams for the various hierarchical lattices are obtained from the renormalization-group flows of the probability distributions. Each phase has a corresponding sink, namely a completely stable fixed distribution. The boundaries between phases flow to unstable fixed distributions, analysis of which yields the order of the phase transition and the values of the critical exponents of second- and higher-order transitions. All the phase diagrams are plotted in terms antiferromagnetic

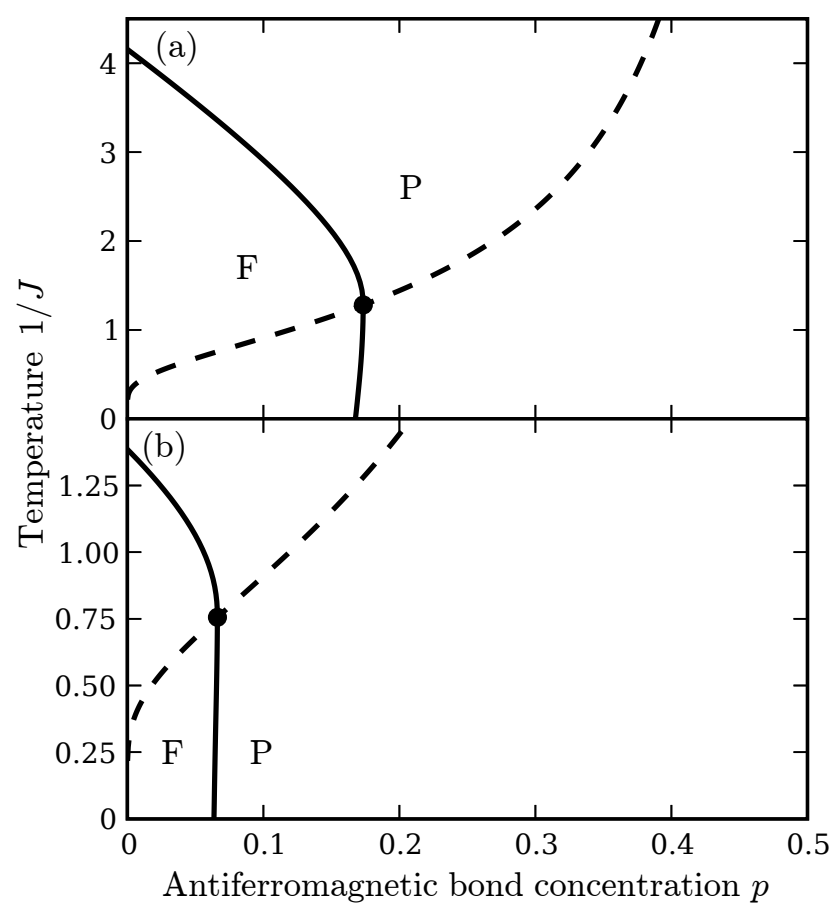

FIG. 4: Phase diagrams for the two hierarchical lattices in Fig. 1, with the solid lines indicating second-order phase transitions between the ferromagnetic $(\mathrm{F})$ and paramagnetic $(\mathrm{P})$ phases. In each diagram the multicritical point, separating two different types of second-order boundary, is marked by a dot, and the Nishimori symmetry line is drawn dashed. The phase diagrams were calculated with 40,000 probability bins, except for the vicinity of the multicritical points, where for higher precision 1,000,000 probability bins were used.

bond concentration $p$ versus temperature $1 / J$. The diagrams are symmetric around $p=1 / 2$, with the ferromagnetic phase in the $p<1 / 2$ half-space mapping onto the antiferromagnetic phase in the $p>1 / 2$ half-space. Thus in the figures only the $p<1 / 2$ portions are shown.

Fig. 4(a) and (b) show the phase diagrams for the dual pair of hierarchical lattices in Fig. 1(a) and (b) respectively. The phase structure of both diagrams is topologically identical to that of the $d=2$ Ising spin-glass on a square lattice, which is only natural considering that the $d=2, b=3$ Migdal-Kadanoff recursion relations are exact on these hierarchical lattices. [6] The $p=0$ transition temperatures of the two models are related by the duality algebra [29]

$$
\sinh \left(2 J_{1 c}\right) \sinh \left(2 J_{2 c}\right)=1,
$$

which is also true for the two other pairs of mutually dual hierarchical models. Furthermore, the $p=0$ transition temperatures in Fig. 4(a) and (b) are related [10] by

$$
J_{1 c}^{-1}=b^{d-1} J_{2 c}^{-1},
$$




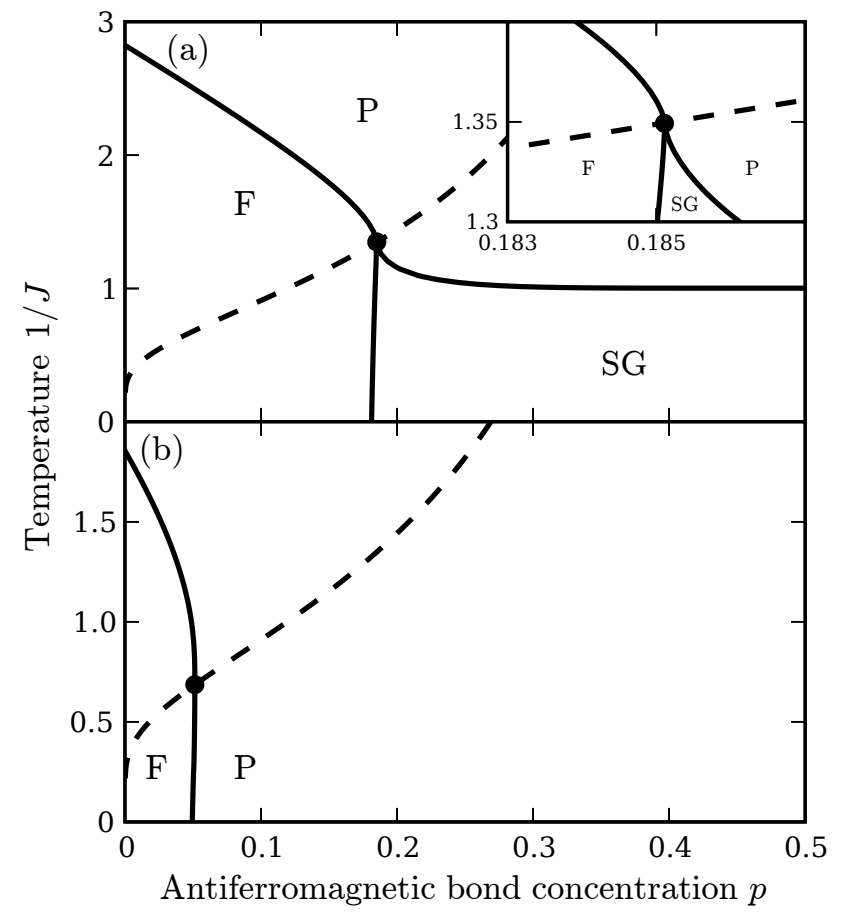

FIG. 5: Phase diagrams for the two hierarchical lattices in Fig. 2, with the solid lines indicating second-order phase transitions between the ferromagnetic $(\mathrm{F})$, paramagnetic $(\mathrm{P})$, and spin-glass (SG) phases. In each diagram the multicritical point is marked by a dot, and the Nishimori symmetry line is drawn dashed. The phase diagrams were calculated with 40,000 probability bins (250,000 bins for the inset in the top figure), except for the vicinity of the multicritical points, where for higher precision $10^{6}$ and $2.5 \times 10^{9}$ probability bins were used in (a) and (b) respectively.

since the mappings of the interaction constant in the repetition of renormalization-group transformations differs only by an initial bond strengthening by a factor of $b^{d-1}$; note that Eq. (19) does not apply to $0<p<1$, since there the bond-moving is not a mere multiplicative strengthening, but a $\left(b^{d-1}\right)$-fold convolution of the probability distributions that alters this distribution in a non-simple way. Eq. (19) is also not applicable to the two other pairs of mutually dual models, since the repetition of renormalization-group transformations are not differentiated by only a preliminary bond-moving.

In each of Fig. 4(a) and (b), a ferromagnetic phase at low temperatures and low $p$ is separated from the disordered paramagnetic phase by two second-order phase boundaries, meeting at a multicritical point. (In a narrow neighborhood of all multicritical points in our results, reentrance is observed: paramagnetic, then ferromagnetic, then paramagnetic or spin-glass phases are encountered as temperature is lowered at fixed $p$.) The two second-order boundaries flow to distinct unstable probability distributions with different critical exponents, con-

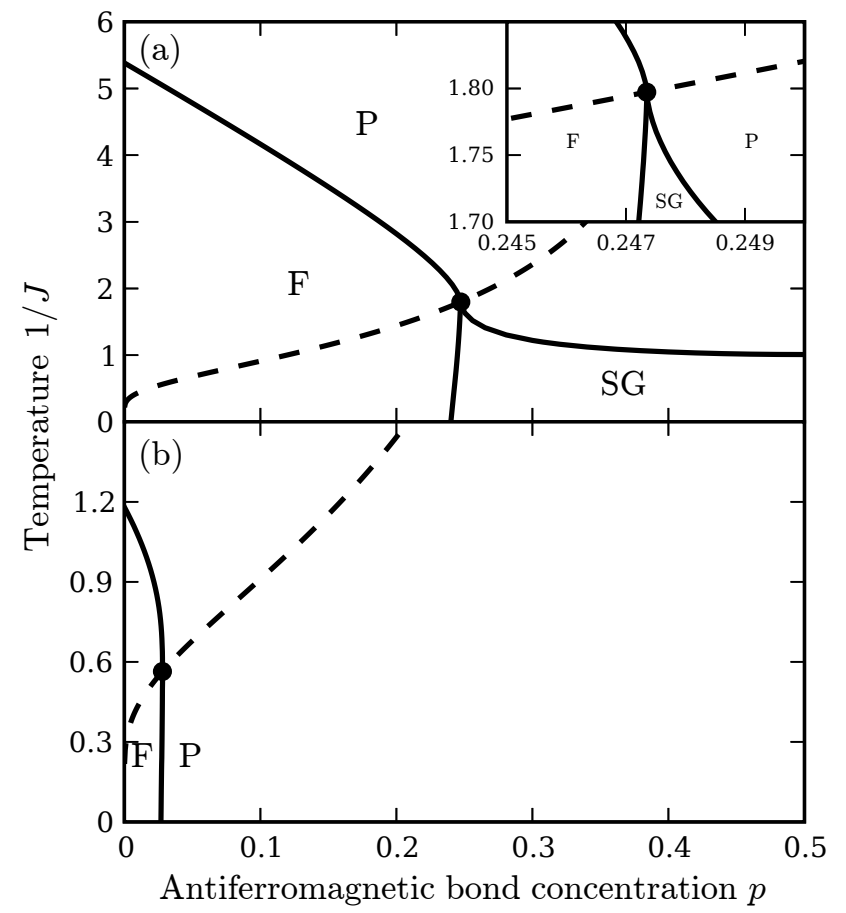

FIG. 6: Phase diagrams for the two hierarchical lattices in Fig. 3, with the solid lines indicating second-order phase transitions between the ferromagnetic $(\mathrm{F})$, paramagnetic $(\mathrm{P})$, and spin-glass (SG) phases. In each diagram the multicritical point is marked by a dot, and the Nishimori symmetry line is drawn dashed. The phase diagrams were calculated with 40,000 probability bins (250,000 bins for the inset in the top figure), except for the vicinity of the multicritical points, where for higher precision $2.5 \times 10^{7}$ and $2.25 \times 10^{8}$ probability bins were used in (a) and (b) respectively.

stituting a strong violation of universality [17] and consistent with the prediction, generally, of the absence of first-order transition under quenched randomness in $d=2$. [30] As expected from symmetry considerations, the multicritical points fall [13, 14, 15] precisely on the Nishimori line 1] as seen in Table I. As also seen in Table I, $H\left(p_{1 m}\right)+H\left(p_{2 m}\right)=1.0172$, so that the conjecture is realized to a very good approximation.

Fig. 5 shows the phase diagrams for the dual pair of hierarchical lattices in Fig. 2. While Fig. 5(b) has the same phase topology as the diagrams in Fig. 4, being at $d=1.5$ below the spin-glass lower-critical dimension, a different structure occurs in Fig. 5(a). Here the $d=3$, $b=3$ Migdal-Kadanoff relations are exact on the hierarchical lattice, and for low temperatures in the vicinity of $p=1 / 2$ there exists a spin-glass phase. The multicritical point occurs where the ferromagnetic, paramagnetic, and spin-glass phases meet. As expected both multicritical points lie directly on the Nishimori line. From Table I we see that $H\left(p_{1 m}\right)+H\left(p_{2 m}\right)=0.9829$, so that the conjecture is realized to a very good approximation, 


\begin{tabular}{|c|c|c|c|c|c|c|c|}
\hline Figure & $p_{1 m}, J_{1 m}^{-1}$ & $J_{1 N}^{-1}$ & $p_{2 m}, J_{2 m}^{-1}$ & $J_{2 N}^{-1}$ & $H\left(p_{1 m}\right)$ & $H\left(p_{2 m}\right)$ & $H\left(p_{1 m}\right)+H\left(p_{2 m}\right)$ \\
\hline \hline Fig. 4 & $0.1735,1.2810$ & 1.2810 & $0.06620,0.7557$ & 0.7557 & 0.6656 & 0.3516 & 1.0172 \\
\hline Fig. 5 & $0.1851,1.3494$ & 1.3494 & $0.05128,0.68546$ & 0.68545 & 0.6911 & 0.2918 & 0.9829 \\
\hline Fig. 6 & $0.2473,1.7973$ & 1.7973 & $0.02796,0.5636$ & 0.5636 & 0.8070 & 0.1840 & 0.9911 \\
\hline
\end{tabular}

TABLE I: Locations of the multicritical points in the phase diagrams of Figs. 4-6 (corresponding to the hierarchical lattices of Figs. 1-3). $J_{i N}^{-1}$ is the value calculated from $p_{i m}$ using Eq. (11) for the Nishimori line and turns out equal to $J_{i m}^{-1}$, for both $i=1$ and 2 . The quantities $H\left(p_{i m}\right)$ that enter the conjecture and their sums are also given.

even when the mutually dual models belong to different dimensionalities $d$ and have different phase diagram topologies at the multicritical points of the conjecture.

The phase diagram structures in Fig. 6, corresponding to the dual pair of hierarchical lattices in Fig. 3, are similar to those of Fig. 5, illustrating dimensions above and below the spin-glass lower-critical dimension. Again the multicritical points for both cases lie directly on the Nishimori line. In this case $H\left(p_{1 m}\right)+H\left(p_{2 m}\right)=0.9911$, and the conjecture is realized to a very good approximation, again for mutually dual models belonging to different dimensionalities $d$ and having different phase diagram topologies at the multicritical points of the conjecture.

Thus, we find that for all three mutually dual pairs of hierarchical lattices, the conjecture relating the locations of the multicritical points is satisfied to a very good approximation. This is all the more remarkable, since, as seen in Table I, the contributions of $H\left(p_{1 m}\right)$ and $H\left(p_{2 m}\right)$ to the conjecture are strongly asymmetric. However, it should be noted that $(1.0172,0.9829,0.9911)$, while being very close to 1 , are different from integer 1 . In our numer- ical implementation of the convolutions of the probability distributions, the results have converged to the precision of the digits shown in Table I. Further increase of the already very large number of probability bins does not change the entries in the table. Further tests of the conjecture, using other systems, would be very useful. Similar to our current study, the use of hierarchical lattices to study phenomena linked to mutually dual lattices, e.g., Ref. [31], would also be very useful.

\section{Acknowledgments}

We thank H. Nishimori and K. Takeda for useful correspondance. This research was supported by the Scientific and Technical Research Council of Turkey (TÜBITAK) and by the Academy of Sciences of Turkey. MH gratefully acknowledges the hospitality of the Feza Gürsey Research Institute and of the Physics Department of Istanbul Technical University.
[1] H. Nishimori, Prog. Theor. Phys. 66, 1169 (1981).

[2] H. Nishimori and K. Nemoto, J. Phys. Soc. Japan 71, 1198 (2002).

[3] J.-M. Maillard, K. Nemoto, and H. Nishimori, J. Phys. A: Math. Gen. 36, 9799 (2003).

[4] K. Takeda and H. Nishimori, Nucl. Phys. B 686, 377 (2004).

[5] K. Takeda, T. Sasamoto, and H. Nishimori, J. Phys. A: Math. Gen. 38, 3751 (2005).

[6] A.N. Berker and S. Ostlund, J. Phys. C 12, 4961 (1979).

[7] M. Kaufman and R.B. Griffiths, Phys. Rev. B 24, 496 (1981).

[8] M. Kaufman and R.B. Griffiths, Phys. Rev. B 30, 244 (1984).

[9] M. Kaufman and D. Andelman, Phys. Rev. B 29, 4010 (1984).

[10] M. Kaufman, Phys. Rev. B 30, 413 (1984).

[11] C. Itzykson and J.M. Luck, Proceedings of the Brasov International Summer School (1984).

[12] H. Ottavi and G. Albinet, J. Phys. A 20, 2961 (1987).

[13] P. Le Doussal and A. Georges, Yale University Report No. YCTP-P1-88 (1988).

[14] P. Le Doussal and A.B. Harris, Phys. Rev. Lett. 61, 625 (1988).

[15] E.J. Hartford and S.R. McKay, J. Appl. Phys. 70, 6068 (1991).
[16] S.R. McKay, A.N. Berker, and S. Kirkpatrick, Phys. Rev. Lett. 48, 767 (1982).

[17] G. Migliorini and A.N. Berker, Phys. Rev. B 57, 426 (1998).

[18] D. Andelman and A.N. Berker, Phys. Rev. B 29, 2630 (1984).

[19] A. Falicov, A.N. Berker, and S.R. McKay, Phys. Rev. B 51, 8266 (1995).

[20] E. Domany, S. Alexander, D. Bensimon, and L.P. Kadanoff, Phys. Rev. B 28, 3110 (1983).

[21] J.-M. Langlois, A.-M.S. Tremblay, and B.W. Southern, Phys. Rev. B 28, 218 (1983).

[22] R.B. Stinchcombe and A.C. Maggs, J. Phys. A 19, 1949 (1986).

[23] T.A.S. Haddad, S.T.R. Pinho, and S.R. Salinas, Phys. Rev. E 61, 3330 (2000).

[24] J.-X. Le and Z.R. Yang, Phys. Rev. E 69, 066107 (2004).

[25] R.A. da Silveira and J.-P. Bouchaud, Phys. Rev. Lett. 93, 015901 (2004).

[26] A.A. Migdal, Zh. Eksp. Teor. Fiz. 69, 1457 (1975) [Sov. Phys. JETP 42, 743 (1976)].

[27] L.P. Kadanoff, Ann. Phys. (N.Y.) 100, 359 (1976).

[28] A. Erbaş, A. Tuncer, B. Yücesoy, and A.N. Berker, Phys. Rev. E 72, 026129 (2005).

[29] I. Syozi, in Phase Transitions and Critical Phenomena, C. Domb and M.S. Green, eds. (Academic, London, 1972), 
vol.1, pp.270-329.

[30] K. Hui and A.N. Berker, Phys. Rev. Lett. 62, 2507 (1989).
[31] F. Camia and C.M. Newman, J. Stat. Phys 114, 1199 (2004). 